\title{
A faunistic study on the braconid wasps (Hymenoptera: Braconidae) of Iran
}

\author{
Najmeh Samin ${ }^{1}$, Juana María Coronado-Blanco ${ }^{2}$, Afshin Hosseini $^{3}$, \\ MaXimilian Fischer ${ }^{4} \&$ Hamid SaKenin ChelaV ${ }^{5}$
}

\author{
${ }^{1}$ Young Researchers and Elite Club, Science and Research Branch, Islamic Azad University, Tehran, Iran; \\ email: n_samin63@yahoo.com \\ ${ }^{2}$ Facultad de Ingeniería y Ciencias, Universidad Autónoma de Tamaulipas, Centro Universitario, Cd. \\ Victoria, Tamaulipas, Mexico \\ ${ }^{3}$ Department of Biology, Yadegar- e- Imam Khomeini (RAH) Shahre Rey Branch, \\ Islamic Azad University, Tehran, Iran \\ ${ }^{4}$ Naturhistorisches Museum, 2. Zoologische Abteilung, A-1010 Wien, Austria \\ ${ }^{5}$ Department of Plant Protection, Qaemshahr Branch, Islamic Azad University, Mazandaran, Iran
}

Samin, N., Coronado-Blanco J. M., Hosseini, A., Fischer, M. \& Chelav, H. S.: A faunistic study on the braconid wasps (Hymenoptera: Braconidae) of Iran.

Abstract: Twenty-three species of Braconidae (Hymenoptera) within eight subfamilies, Agathidinae, Alysiinae, Blacinae, Brachistinae, Braconinae, Cheloninae, Euphorinae, Macrocentrinae, Microgastrinae and Neoneurinae are recorded for the fauna of Iran. Five species, Macrocentrus grandii Goidanich, 1937 (Macrocentrinae), Apanteles elpis Nixon, 1973, Apanteles urgo Nixon, 1965, Microplitis sofron Nixon, 1970, and Protapanteles aliphera (Nixon, 1973) (Microgastrinae) are newly recorded from Iran.

Keywords: Braconidae, species diversity, parasitoid, Iran

\section{Introduction}

Braconidae is a family of parasitoid wasps and one of the richest families of insects with more than 21,000 described species worldwide (YU et al. 2016). Braconid wasps are very powerful and important biological control agent in most ecosystems (GoDFRAY 1994). Most braconids are primary parasitoids of Coleoptera, Diptera, and Lepidoptera, but also some hemimetabolous insects like aphids, Heteroptera or Embiidina (WHARTON 1993, QUiCKE 2015).

The fauna of Iranian Braconidae has been studied rather well (e.g., GANDALLAH \& GHAHARI 2013a, b, 2015, 2017; BARAHOEI et al. 2014; GANDALlaH et al. 2015a, b, 2016a, b; Gandallah \& Ghahari 2016; Farahani et al. 2016; Ghahari 2016; BEYARSLAN et al. 2017; SAMIN et al. 2018a, b), but since Iran is a large country comprises various ecosystems, several new species are expected to be discovered. The objective of this faunistic paper is to study of distribution of 23 braconid species and five new country records. 


\section{Material and methods}

The specimens of this investigation were collected from different regions of Iran by Malaise traps and sweeping nets, and a few by rearing of the hosts in incubator. The specimens were identified by the fourth author, C. van Achterberg (National Natuurhistorisch Museum) and the late J. Papp (Hungarian Natural History Museum). Classification, nomenclature, distribution and host records of Braconidae suggested by Yu et al. (2016) have been followed.

\section{Results}

In total, 23 species of Braconidae within eight subfamilies, Agathidinae (one species), Alysiinae (one species), Blacinae (one species), Brachistinae (four species in two genera), Braconinae (three species in two genera), Cheloninae (two species in one genus), Euphorinae (two species in two genera), Macrocentrinae (two species in one genus), Microgastrinae (seven species in four genera) and Neoneurinae (one species) are recorded for the fauna of Iran.

\section{Subfamily Agathidinae Haliday, 1833 \\ Genus Therophilus Wesmael, 1837}

Therophilus tegularis (Thomson, 1895)

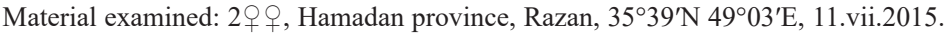

General distribution: Bulgaria, France, Germany, Hungary, Italy, Netherlands, Norway, Poland, Sweden, Turkey, United Kingdom, former Yugoslavia.

\section{Subfamily Alysiinae Leach, 1815 Genus Aspilota Förster, 1862}

Aspilota fuscicornis (Haliday, 1838)

Material examined: 1 , , Khuzestan province, Shadegan, $30^{\circ} 66^{\prime} \mathrm{N} 48^{\circ} 67^{\prime} \mathrm{E}$, 4.v.2014.

General distribution: Austria, Belgium, former Czechoslovakia, Faeroe Islands, Finland, Germany, Greece, Hungary, Iceland, Ireland, Italy, Korea, Lithuania, Madeira Islands, Mongolia, Netherlands, Poland, Romania, Russia, Spain, Sweden, United Kingdom.

\section{Subfamily Blacinae Förster, 1863 \\ Genus Blacus Nees, 1819}

Blacus (Blacus) forticornis Haeselbarth, 1973

Material examined: 1 \% , West Azarbaijan province, Shahin-Dezh, 36 $68^{\prime} \mathrm{N} 46^{\circ} 56^{\prime} \mathrm{E}, 26 . i x .2015$.

General distribution: Czech Republic, Denmark, Hungary, Poland, Russia, Turkey, United Kingdom.

\section{Subfamily Brachistinae Förster, 1863 \\ Genus Schizoprymnus Förster, 1862}

Schizoprymnus (Schizoprymnus) elongatus (Szépligeti, 1898)

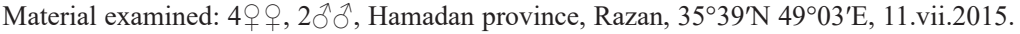

General distribution: Afghanistan, Armenia, Azerbaijan, Czech Republic, former Czechoslovakia, Georgia, Kazakhstan, Lithuania, Moldova, Turkey. 
Schizoprymnus (Schizoprymnus) excisus (Snoflák, 1953)

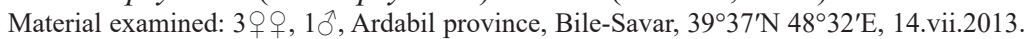

General distribution: Armenia, Hungary, Russia, Turkey, Ukraine, former Yugoslavia.

Schizoprymnus (Schizoprymnus) pullatus (Dahlbom, 1833)

Material examined: 2 우, $1{ }^{\jmath}$, Khuzestan province, Shoosh, $32^{\circ} 19^{\prime} \mathrm{N} 48^{\circ} 24^{\prime} \mathrm{E}$, 3.v.2014.

General distribution: Former Czechoslovakia, Germany, Hungary, Kazakhstan, Moldova, Russia, Turkey, Uzbekistan.

\section{Genus Triaspis Haliday, 1835}

Triaspis luteipes (Thomson, 1874)

Material examined: 1 , , Fars province, Abadeh, $31^{\circ} 20^{\prime} \mathrm{N} 51^{\circ} 62^{\prime} \mathrm{E}$, 9.vi.2015.

General distribution: Bulgaria, Finland, France, Germany, Greece, Hungary, Italy, Poland, Romania, Spain, Sweden, Switzerland, Ukraine, United Kingdom.

\section{Subfamily Braconinae Nees, 1811 Genus Bracon Fabricius, 1804}

Bracon (Glabrobracon) brevicalcaratus Tobias, 1957

Material examined: 1ㅇ, Ardabil province, Bile-Savar, 39³7'N 48³2'E, 14.vii.2013.

General distribution: Hungary, Kazakhstan, Turkey, Turkmenistan.

Bracon (Habrobracon) viktorovi (Tobias, 1961)

Material examined: 1 을 East Azarbaijan province, Maragheh, $37^{\circ} 23^{\prime} \mathrm{N} 46^{\circ} 24^{\prime} \mathrm{E}$, 21.viii.2013.

General distribution: Cyprus, former Czechoslovakia, Greece, Korea, Russia, Turkey.

Genus Iphiaulax Förster, 1863

Iphiaulax (Iphiaulax) jacobsoni Shestakov, 1927

Material examined: 1 ㅇ, $1{ }^{\AA}$, Ardabil province, Firozabad, $37^{\circ} 59^{\prime} \mathrm{N} 48^{\circ} 24^{\prime} \mathrm{E}$, 12.vii.2013.

General distribution: Israel, Malta, Turkey, Uzbekistan.

\section{Subfamily Cheloninae Förster, 1863 Genus Chelonus Panzer 1806}

Chelonus (Chelonus) mirandus Tobias, 1964

Material examined: $1 \delta^{\Uparrow}$, Zanjan province, Abhar, $36^{\circ} 16^{\prime} \mathrm{N} 49^{\circ} 03^{\prime} \mathrm{E}, 7 . v i .2016$.

General distribution: Hungary, Kazakhstan, Moldova, Mongolia, Poland, Slovakia, Spain.

Chelonus (Chelonus) pannonicus Szépligeti, 1896

Material examined: 2 우, Fars province, Abadeh, $31^{\circ} 20^{\prime} \mathrm{N} 51^{\circ} 62^{\prime} \mathrm{E}, 9 . v i .2015$.

General distribution: China, Croatia, Czech Republic, former Czechoslovakia, Greece, Hungary, Mongolia, Ukraine, former Yugoslavia.

Subfamily Euphorinae Förster, 1863

Genus Leiophron Nees von Esenbeck, 1819

Leiophron (Leiophron) apicalis Haliday, 1833

Material examined: $1 \delta^{\lambda}$, Ardabil province, Aslanduz, $39^{\circ} 44^{\prime} \mathrm{N} 37^{\circ} 42^{\prime} \mathrm{E}, 1$,, $1 \delta^{\lambda}$, 28.vii.2014.

General distribution: Azerbaijan, former Czechoslovakia, France, Germany, Hungary, Netherlands, Poland, United Kingdom, Uzbekistan. 


\section{Genus Perilitus Nees, 1819}

Perilitus dubius (Wesmael, 1838)

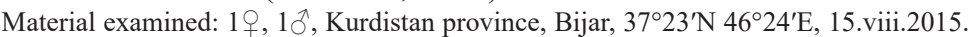

General distribution: Belgium, former Czechoslovakia, Finland, France, Germany, Greece, Hungary, Kazakhstan, Moldova, Netherlands, Poland, Russia, Sweden, Switzerland, United Kingdom.

\section{Subfamily Macrocentrinae Förster, 1863 Genus Macrocentrus Curtis, 1833}

\section{Macrocentrus grandii Goidanich, 1937}

Material examined: $2 \mathrm{o}^{\lambda} \mathrm{o}^{\lambda}$, Golestan province, Kordkoy, $36^{\circ} 41^{\prime} \mathrm{N} 54^{\circ} 12^{\prime} \mathrm{E}$, ex Ostrinia nubilalis (Hübner, 1796) (Lepidoptera: Crambidae) on Zea mays, 23.ix.2013. New record for Iran.

General distribution: Azerbaijan, Bulgaria, Georgia, Germany, Italy, Japan, Korea, Lithuania, Moldova, Russia, Slovakia, United States of America.

Macrocentrus linearis (Nees, 1811)

Material examined: 1 우 $10^{\widehat{ }}$, Ardabil province, Aslanduz, $39^{\circ} 44^{\prime} \mathrm{N} 37^{\circ} 42^{\prime} \mathrm{E}$, ex Malacosoma neustria (Linnaeus, 1758) (Lepidoptera: Lasiocampidae), 28.vii.2014.

General distribution: Eastern Palaearctic, Ethiopian, Europe, Nearctic, Oriental, Western Palaearctic.

\section{Subfamily Microgastrinae Förster, 1863 Genus Apanteles Förster, 1863}

\section{Apanteles elpis Nixon, 1973}

Material examined: $1{ }^{\jmath}$, East Azarbaijan province, Maragheh, $37^{\circ} 23^{\prime} \mathrm{N} 46^{\circ} 24^{\prime} \mathrm{E}$, 14.vii.1999. New record for Iran.

General distribution: Austria, Azerbaijan, Bulgaria, former Czechoslovakia, Finland, Germany, Hungary, Korea, Mongolia, Poland, Slovakia, United Kingdom.

Apanteles litae Nixon, 1972

Material examined: 2 웅, $1{ }^{\top}$, West Azarbaijan province, Mahabad, $36^{\circ} 46^{\prime} \mathrm{N} 45^{\circ} 44^{\prime} \mathrm{E}$, ex Plutella xylostella (Linnaeus, 1758) (Lepidoptera: Plutellidae), 16.viii.2013.

General distribution: Cape Verde Islands, Cyprus, Egypt, Germany, Ghana, Greece, Hungary, Israel, Italy, Jordan, Spain, Switzerland, Turkey, former Yugoslavia.

Apanteles urgo Nixon, 1965

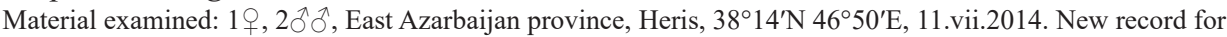
Iran.

General distribution: Azerbaijan, former Czechoslovakia, Greece, Hungary, Mongolia, Turkey.

\section{Genus Microgaster Latreille, 1804}

Microgaster curvicrus Thomson, 1895

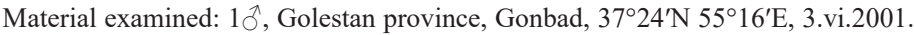

General distribution: Austria, Azerbaijan, Bulgaria, Croatia, Finland, Germany, Hungary, Moldova, Mongolia, Poland, Romania, Russia, Serbia, Sweden, Switzerland, Turkmenistan, United Kingdom, former Yugoslavia.

\section{Genus Microplitis Förster, 1863}

Microplitis fordi Nixon, 1970

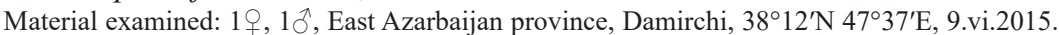

General distribution: Austria, Bulgaria, Germany, Greece, Hungary, Israel, Italy, Jordan, Mongolia, Switzerland, Tunisia, Turkey, United Kingdom. 
Microplitis sofron Nixon, 1970

Material examined: $10^{\circ}$, Qazvin province, Takestan, $36^{\circ} 00^{\prime} \mathrm{N} 49^{\circ} 33^{\prime} \mathrm{E}$, 26.vii.2006. New record for Iran. General distribution: Azerbaijan, Bulgaria, Denmark, Finland, France, Germany, Greece, Greenland, Hungary, Ireland, Italy, Kazakhstan, Netherlands, Norway, Russia, Spain, Sweden, Switzerland, Turkey, United Kingdom.

\section{Genus Protapanteles Ashmead, 1898}

\section{Protapanteles aliphera (Nixon, 1973)}

Material examined: 2 우, Guilan province, Astara, $38^{\circ} 20^{\prime} \mathrm{N} 48^{\circ} 46^{\prime} \mathrm{E}$, 7.vii.2014. New record for Iran. General distribution: Armenia, Azerbaijan, former Czechoslovakia, Finland, France, Georgia, Germany, Greece, Hungary, Israel, Netherlands, Poland, Romania, Russia, Slovakia, Sweden, Switzerland, United Kingdom.

\section{Subfamily Neoneurinae Bengtsson, 1918 Genus Elasmosoma Ruthe, 1858}

Elasmosoma berolinense (Ruthe, 1858)

Material examined: $1{ }^{\top}$, Khuzestan province, Shoosh, $32^{\circ} 19^{\prime} \mathrm{N} 48^{\circ} 24^{\prime} \mathrm{E}$, 3.v. 2014.

General distribution: Albania, Austria, Bulgaria, Canada, China, Croatia, former Czechoslovakia, Denmark, Estonia, Finland, France, Germany, Greece, Hungary, Iran, Italy, Japan, Kazakhstan, Luxemburg, Macedonia, Moldova, Mongolia, Netherlands, Poland, Russia, Slovakia, Slovenia, Spain, Sweden, Tajikistan, Turkey, United States of America, United Kingdom, former Yugoslavia.

\section{Acknowledgements}

The authors are grateful to C. van Achterberg (the Netherlands) and J. Papp (Hungary) for identification of some materials. This research was supported by Islamic Azad University (Young Researchers and Elite Club, Science and Research Branch, Qaemshahr and Shahre Rey Branches). 


\section{References}

Barahoei, H., Rakhshani, E., Nader, E., Starý, P., Kavallieratos, N. G., Tomanović, Z. \& Mehrparvar, M. 2014: Checklist of Aphidiinae parasitoids (Hymenoptera: Braconidae) and their host aphid associations in Iran. - Journal of Crop Protection 3(2): 199-232.

Beyarslan, A., Gadallah, N. S. \& Ghahari, H. 2017: An annotated catalogue of the Iranian Microtypinae and Rogadinae (Hymenoptera: Braconidae). - Zootaxa 4291(1): 099-116.

Farahani, S., TAlebi, A. A. \& Rakhshani, E. 2016: Iranian Braconidae (Insecta: Hymenoptera: Ichneumonoidea): diversity, distribution and host association. - Journal of Insect Biodiversity and Systematics 2(1): 1-92.

Gadallah, N. S. \& Ghahari, H. 2013a: An annotated catalogue of the Iranian Agathidinae and Brachistinae (Hymenoptera: Braconidae). - Linzer biologische Beiträge 45/2: 1873-1901.

Gadallah, N. S. \& Ghahari, H. 2013b: An annotated catalogue of the Iranian Cheloninae (Hymenoptera: Braconidae). - Linzer biologische Beiträge 45/2: 1921-1943.

Gadallah, N. S. \& Ghahari, H. 2015: An annotated catalogue of the Iranian Braconinae (Hymenoptera: Braconidae). - Entomofauna 36: 121-176.

Gadallah, N. S., Ghahari, H., Fischer, M. \& Peris-Felipo, F. J. 2015a: An annotated catalogue of the Iranian Alysiinae (Hymenoptera: Braconidae). - Zootaxa 3974(1): 1-28.

Gadallah, N. S., Ghahari, H. \& Peris-Felipo, F. J. 2015b: Catalogue of the Iranian Microgastrinae (Hymenoptera: Braconidae). - Zootaxa 4043(1): 1-69.

Gadallah, N. S., Ghahari, H., Peris-Felipo, F. J. \& Fischer, M. 2016a: Updated checklist of Iranian Opiinae (Hymenoptera: Braconidae). - Zootaxa 4066(1): 1-40.

Gadallah, N. S., Ghahari, H. \& van AchterberG, C. 2016b: An annotated catalogue of the Iranian Euphorinae, Gnamptodontinae, Helconinae, Hormiinae and Rhysipolinae (Hymenoptera: Braconidae). Zootaxa 4072 (1): 1-38.

Gadallah, N. S. \& Ghahari, H. 2016: An updated checklist of the Iranian Miracinae, Pambolinae and Sigalphinae (Hymenoptera: Braconidae). - Orsis 30: 51-61.

Gadallah, N. S. \& Ghahari, H. 2017: An annotated catalogue of the Iranian Doryctinae and Exothecinae (Hymenoptera: Braconidae). - Transactions of the American Entomological Society 143: 669-691.

GHAHARI, H. 2016: Five new records of Iranian Braconidae (Hymenoptera: Ichnemonoidea) for Iran and annotated catalogue of the subfamily Homolobinae. - Wuyi Science Journal 32: 35-43.

Godfray, H. C. J. 1994: Parasitoids, behavioral and evolutionary ecology. - Princeton University Press. 473 pp.

Quicke, D. L. J. 2015: The braconid and ichneumonid parasitoid wasps: Biology, systematics, evolution and ecology. - Wiley Blackwell, Chichester. 688 pp.

Samin, N., Coronado-Blanco, J.M., Kavallieratos, N.G., Fischer, M. \& Sakenin, H. 2018a: Recent findings on Braconidae (Hymenoptera: Ichneumonoidea) of Iran with an updated checklist. - Acta Biologica Turcica 31(4): 160-173.

Samin, N., Coronado-Blanco, J. M., Fischer, M., van Achterberg, C., Sakenin, H. \& Davidian, E. 2018b: Updated checklist of Iranian Braconidae (Hymenoptera: Ichneumonoidea) with twenty-three new records. - Natura Somogyiensis 32: 21-36.

Wharton, R. A. 1993. Bionomics of the Braconidae. - Annual Review of Entomology 38: 121-143.

Yu, D. S., van Achterberg, K. \& Horstmann, K. 2016: World Ichneumonoidea 2011. Taxonomy, Biology, Morphology and Distribution. - Taxapad.com. Canada. 